\title{
Deep UV Lithography for Planar Photonic Crystal Structures
}

\author{
Wim Bogaerts ${ }^{1}$, Pieter Dumon ${ }^{1}$, Joris Van Campenhout ${ }^{1}$, Vincent Wiaux ${ }^{2}$, Johan Wouters ${ }^{2}$, \\ Stephan Beckx ${ }^{2}$, Dirk Taillaert ${ }^{1}$, Bert Luyssaert ${ }^{1}$, Dries Van Thourhout ${ }^{1}$, Roel Baets ${ }^{1}$ \\ ${ }^{1}$ Ghent University - IMEC, Dept. of Information Technology, Sint-Pietersnieuwstraat 41 \\ 9000 Gent, BELGIUM, roel.baets@intec.UGent.be \\ ${ }^{2}$ IMEC vzw, Silicon Process Technology Division, Kapeldreef 75, 3001 Leuven, BELGIUM
}

Abstract We demonstrate deep UV lithography at $248 \mathrm{~nm}$ to be a useful fabrication tool for nanophotonics, including photonic crystals, operating at telecom wavelengths. The structures are of high quality, and first results show low propagation losses.

\section{Introduction}

Photonic Crystals are photonic structures with periodic variations of the refractive index and a wavelength-scale period [1]. This makes the fabrication of 2-D photonic crystals for telecom wavelengths a nontrivial task, especially for commercial applications. Unlike with research, where e-beam lithography is the fabrication method of choice, commercial components need a fabrication technology that can deliver large throughput as well as accuracy. Therefore an alternative is needed if one considers massfabricated components based on photonic crystals.

Deep UV lithography (DUV) is an optical lithography technology for wavelengths of $248 \mathrm{~nm}$ and shorter and is already widely used for the fabrication of advanced CMOS components. We have performed experiments for the fabrication of PhC-based structures in silicon-on-insulator with 248nm DUV lithography and measured transmission through waveguides and bends. In order to get acceptable waveguide losses, modifications of the standard fabrication processes were needed.

\section{Fabrication process}

The fabrication was carried out at IMEC using deep UV lithography at 248nm on Silicon-on-insulator [2]. The features are defined with a stepper on an 8 " SOI wafer ( $220 \mathrm{~nm}$ top Silicon with $1 \mu \mathrm{m}$ of oxide), and the illumination conditions can be varied for each die. This makes it possible to make a wide variety of structure sizes on a single wafer. After lithography, the wafers then go through a Silicon etch, and optionally through an additional etch about $800 \mathrm{~nm}$ into the oxide.

The top row of Figure 1 contains deeply etched structures, with both Silicon and oxide etch. The bottom row contains the same structures, but with only the Silicon etch. The deeply etched structures have significantly more sidewall roughness, reducing the performance of the waveguide components [3].
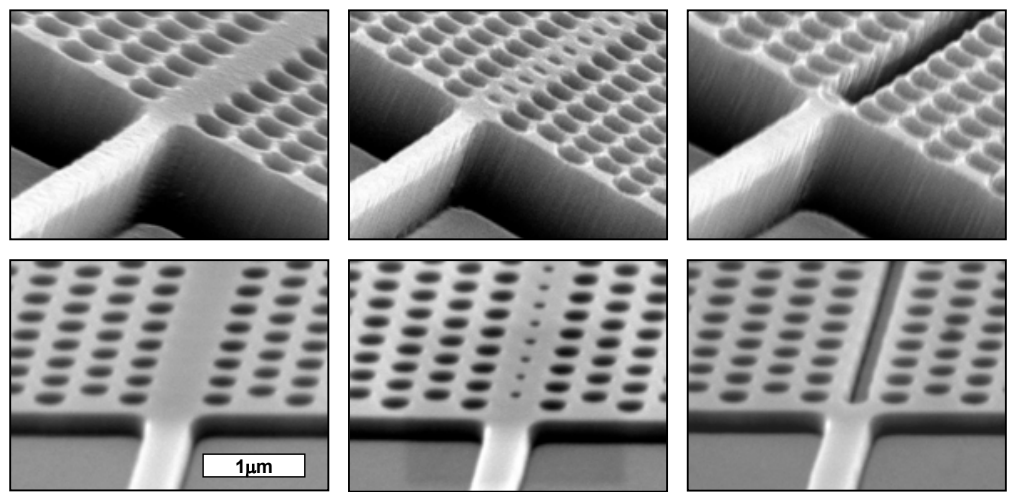

Figure 1: Photonic crystal waveguides fabricated with deep UV lithography. Top row: deeply etched, through the top Silicon and the buried oxide. Bottom row: Silicon-only etch. 


\section{Fabricated structures}

We have fabricated a variety of structures using deep UV lithography, some of which are illustrated in Figure 2. At the time of writing, we have measured propagation losses $20 \mathrm{~dB} / \mathrm{mm}$ in deeply etched photonic crystal waveguides and $30 \mathrm{~dB} / \mathrm{mm}$ in deeply etched photonic wires of $500 \mathrm{~nm}$ wide. For broader wires, $(600 \mathrm{~nm}$ width) this loss drops to $6 \mathrm{~dB} / \mathrm{mm}$. When we etch only the top Silicon layer, the sidewall roughness in these structures is dramatically reduced. For the photonic wires with Silicon-only etch, this results in a propagation loss as low as $0.24 \mathrm{~dB} / \mathrm{mm}$. At the time of writing, photonic crystal waveguides with a Silicon-only etch have not yet been characterised, but results will be presented at the conference.

Next to waveguide structures, we have also fabricated grating structures for coupling light to a vertically butt-coupled single-mode fibre. This can be done with a 1-D grating, coupling one fibre polarisation [4], or with a 2-D grating, coupling each fibre polarisation into a TE waveguide mode in orthogonal waveguides [5]. The fibre couplers have an experimental efficiency of $16 \%$ in coupling the fibre mode to a $10 \mu \mathrm{m}$ wide ridge waveguide in SOI. To squeeze the light further down to the core of a nanophotonic waveguide, we have designed interference-based spotsize convertors, like the one in the third part of Figure 2. Theoretical studies have shown coupling efficiencies of more than $90 \%$.
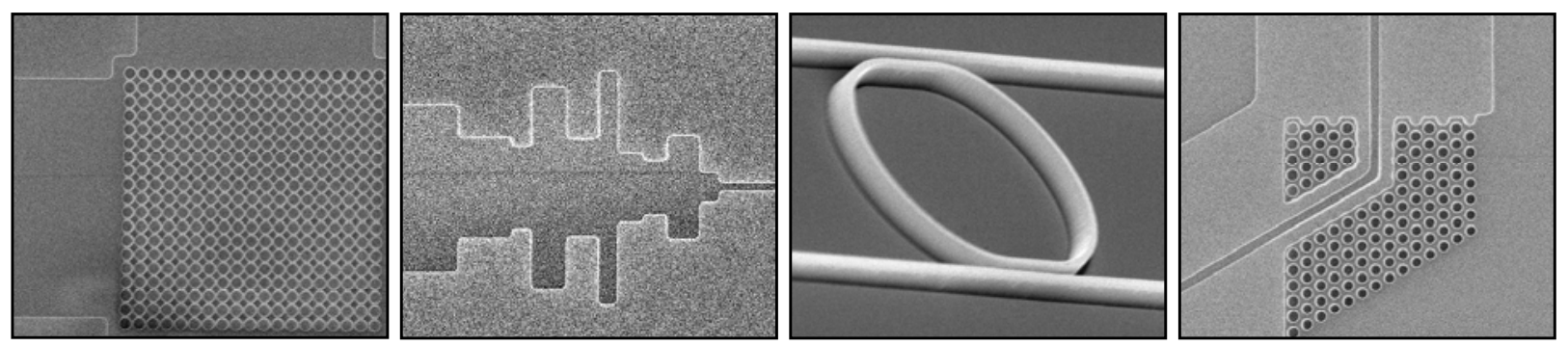

Figure 2: Various structures fabricated with deep UV lithography. Left to right: A square grating for vertical fibre coupling and polarisation splitting, an interference-based spot-size convertor. A deeply etched racetrack resonator, A photonic wire bend embedded in a photonic crystal.

\section{Conclusions}

We demonstrate a wide variety of SOI nanophotonic structures fabricated with deep UV lithography. For deeply etched structures, the waveguide losses are limited to several $\mathrm{dB} / \mathrm{mm}$ due to significant sidewall roughness. These losses can be reduced dramatically by omitting the oxide etch, as we have already measured on ridge waveguides and photonic wires.

\section{Acknowledgements}

Part of this work was done in the context of the IST-PICCO project supported by the European Union. Part of this work was carried out in the context of the Belgian IAP PHOTON network

Wim Bogaerts and Pieter Dumon thank the Flemish Institute for the industrial advancement of scientific and technological Research (IWT) for a specialisation grant.

Joris Van Campenhout was supported by the Flemish Fund for Scientific Research (FWO - Vlaanderen) through a doctoral fellowship.

The authors would like to thank Diziana Vangoidsenhoven for the wafer exposures, and Rudi de Ruyter and Johan Mees for their work on the mask design.

\section{References}

[1] E. Yablonovitch, Phys. Rev. Lett. 58 (1987), 2059

[2] W. Bogaerts et al, IEEE. J. Sel. Top. Quant. Electron. 8(4), p.928

[3] W. Bogaerts et al, Opt. Lett. 28(9), p. 689

[4] D. Taillaert et al., IEEE. J. Quant. Electron. 38(7), p.949

[5] D. Taillaert et al., Proc. ECIO 2003, p. PD:5-8 